\title{
A NOTE ON THE AREA OF A NONPARAMETRIC SURFACE
}

HARRY D. HUSKEY

1. Introduction. Recently, Besicovitch $[1]^{1}$ has exhibited a surface of the form $z=f(x, y)$ for which sequences of inscribed polyhedra, corresponding to triangulations into nearly isosceles right triangles, do not converge in area to the area of the surface. On the other hand, for surfaces defined by functions $f(x, y)$ which are absolutely continuous in the sense of Tonelli this paper shows (see Theorem I) that in a statistical sense sequences of inscribed polyhedra that arise from triangulations which are successive refinements and which consist of right triangles of any preassigned shape (exactly isosceles, for example) will converge in area to the area of the surface. For surfaces of a more general class (defined by functions which are absolutely continuous in the sense of Young, see below) analogous but somewhat weaker theorems (see Theorems II and III) are proved.

In order to state the results more precisely let us consider the following definitions. Let $Q_{0}$ denote the unit square $0 \leqq x \leqq 1,0 \leqq y \leqq 1$. Let $S$ denote the continuous surface defined by the continuous function $z=f(x, y)$ for $-\infty<x<+\infty,-\infty<y<+\infty$. Suppose that $f(x, y)$ is periodic of period one in $x$ and in $y$. The results of this paper are valid for a continuous function defined only on $Q_{0}$ since by extension of definition and a suitable change of scale (see Saks [8, p. 170]) the above conditions can be satisfied.

Let $D_{n}{ }^{0}(u, v)$, for $0 \leqq u \leqq 1$ and $0 \leqq v \leqq 1$, denote a subdivision of $Q_{0}$ into rectangles formed by the lines $x=u+i / n$ and $y=v+i / n$ where $i$ takes on all integral values (positive, negative, and zero) which give lines across $Q_{0}$. Let $\left(x^{\prime}, y^{\prime}\right),\left(x^{\prime}, y^{\prime \prime}\right),\left(x^{\prime \prime}, y^{\prime}\right)$, and $\left(x^{\prime \prime}, y^{\prime \prime}\right)$ denote the vertices of a generic rectangle with $x^{\prime}<x^{\prime \prime}$ and $y^{\prime}<y^{\prime \prime}$. Let

$$
\begin{aligned}
T_{1}\left(f ; x^{\prime}, y^{\prime}\right)=\frac{1}{2}\left\{1+\left[\frac{f\left(x^{\prime \prime}, y^{\prime}\right)-f\left(x^{\prime}, y^{\prime}\right)}{x^{\prime \prime}-x^{\prime}}\right]^{2}\right. \\
\left.+\left[\frac{f\left(x^{\prime}, y^{\prime \prime}\right)-f\left(x^{\prime}, y^{\prime}\right)}{y^{\prime \prime}-y^{\prime}}\right]^{2}\right\}^{1 / 2},
\end{aligned}
$$

$$
\begin{aligned}
T_{2}\left(f ; x^{\prime}, y^{\prime}\right)=\frac{1}{2}\left\{1+\left[\frac{f\left(x^{\prime \prime}, y^{\prime \prime}\right)-f\left(x^{\prime}, y^{\prime \prime}\right)}{x^{\prime \prime}-x^{\prime}}\right]^{2}\right. \\
\left.+\left[\frac{f\left(x^{\prime \prime}, y^{\prime \prime}\right)-f\left(x^{\prime \prime}, y^{\prime}\right)}{y^{\prime \prime}-y^{\prime}}\right]^{2}\right\}^{1 / 2} .
\end{aligned}
$$

Received by the editors March 7, 1946.

1 Numbers in brackets refer to the references cited at the end of the paper. 
Now $\left(x^{\prime \prime}-x^{\prime}\right)\left(y^{\prime \prime}-y^{\prime}\right) T_{i}\left(f ; x^{\prime}, y^{\prime}\right), i=1,2$, are the areas of two triangles inscribed in the surface $S: z=f(x, y)$ and defined over the rectangle $x^{\prime} \leqq x \leqq x^{\prime \prime}, y^{\prime} \leqq y \leqq y^{\prime \prime}$. Thus

$$
\begin{aligned}
A\left[P_{0}^{n}(f ; u, v)\right] & =\sum_{D_{n}^{0}(u, v)}\left[T_{1}\left(f ; x^{\prime}, y^{\prime}\right)+T_{2}\left(f ; x^{\prime}, y^{\prime}\right)\right]\left(x^{\prime \prime}-x^{\prime}\right)\left(y^{\prime \prime}-y^{\prime}\right),
\end{aligned}
$$

where the summation is over the vertices $\left(x^{\prime}, y^{\prime}\right)$ of $D_{n}{ }^{0}(u, v)$, is the area of a polyhedron $P_{0} n(f ; u, v)$ inscribed in $S$ and defined over $Q_{0}$.

The Lebesgue area of a surface $S: z=f(x, y),(x, y) \in Q_{0}$, can be defined as follows:

$$
A(S)=\text { g.l.b. } \lim _{n} \inf E\left(P_{n}\right)
$$

where $E\left(P_{n}\right)$ is the area of the polyhedron $P_{n}$ in the elementary sense and the greatest lower bound is taken over all sequences of polyhedra $P_{n}: z=p_{n}(x, y),(x, y) \in Q_{0}$, such that $p_{n}(x, y)$ converges uniformly on $Q_{0}$ to $f(x, y)$. We shall assume that the reader is familiar with the definitions of bounded variation in the sense of Tonelli (BVT) and absolute continuity in the sense of Tonelli (ACT) (see, for example, Saks [8, p. 169]). We shall further assume familiarity with the concept of absolute continuity of a function on a Borel set (see Young [9, p. 52]).

Let $f(x, y)$ be continuous and BVT on a rectangle $R: a \leqq x \leqq b$, $c \leqq y \leqq d$. Then we shall say that $f(x, y)$ is absolutely continuous in the sense of Young on $R$, or simply ACY on $R: a \leqq x \leqq b, c \leqq y \leqq d$, if the following conditions hold:

(a) $R=B^{\prime}+B^{\prime \prime}$, where $B^{\prime}$ and $B^{\prime \prime}$ are Borel sets;

(b) for each $x, a \leqq x \leqq b$, let $B_{x}^{\prime}$ be the intersection of $B^{\prime}$ with the segment $x=x, c \leqq y \leqq d . B_{y}^{\prime \prime}$ is defined similarly. For almost every $x$, $f(x, y)$ is absolutely continuous on $B_{x}^{\prime}$ as a function of $y$, and, for almost every $y, f(x, y)$ is absolutely continuous on $B_{y}^{\prime \prime}$ as a function of $x$. The term ACY is justified by the fact that L. C. Young [9] first formulated this condition and made an important application of it.

The following theorems will be proved in this paper.

THEOREM I. If $f(x, y)$ is $A C T$ on $Q_{0}$ then for every increasing sequence $\left\{n_{i}\right\}_{i=1}^{\infty}$ of positive integers such that $n_{i}$ divides $n_{i+1}$ for all $i$ we have

$$
\lim _{i} A\left[P_{0}^{n_{i}}(f ; u, v)\right]=A(S)
$$

for almost every $(u, v) \in Q_{0}$. 
THEOREM II. If $f(x, y)$ is $A C Y$ on $Q_{0}$ then for almost every $(u, v) \in Q_{0}$

$$
\lim _{n} \inf A\left[P_{0}^{n}(f ; u, v)\right]=A(S) \text {. }
$$

THEOREM III. If $f(x, y)$ is $A C Y$ on $Q_{0}$ then for any sequence $\left\{n_{i}\right\}_{i=1}^{\infty}$ of positive integers with $n_{i} \rightarrow+\infty$, there exists a subsequence $\left\{n_{\alpha}\right\}$ of $\left\{n_{i}\right\}$ such that for almost every $(u, v) \in Q_{0}$

$$
\lim _{\alpha} A\left[P_{0}^{n \alpha}(f ; u, v)\right]=A(S) .
$$

In the study of the area of nonparametric surfaces various people have imposed conditions of regularity on the triangulations considered. Inspection of the proofs which follow will show that the subdivision $D_{n}{ }^{0}(u, v)$ may be replaced by a subdivision $D_{m n}{ }^{0}(u, v)$ arising from $m$ intervals in the $x$-direction and $n$ in the $y$-direction, and the theorems analogous to those above but stated for squares $Q(u, v)$ (cf. \$2) would still be valid. In order to give theorems valid on $Q_{0}$ Lemma 1 (cf. §2) requires that $m / n$ and $n / m$ both be bounded (also see Radó [5]).

If in the above definition of the Lebesgue area of a surface $S$ we further require that the polyhedra be inscribed in $S$ we obtain a quantity $A^{*}(S)$, and clearly $A^{*}(S) \geqq A(S)$. The question of when $A(S)=A^{*}(S)$ has been called the Problem of Geöcze. It has been proved (see Huskey [2], for example) that $A(S)=A^{*}(S)$ if $f(x, y)$ is ACT on $Q_{0}$. Theorem I implies more than this, namely, that almost every sequence constructed as above converges in area to $A(S)$ if $f(x, y)$ is ACT on $Q_{0}$.

The fundamental, and so far unsolved, problem here is whether just continuity of $f(x, y)$ implies $A(S)=A^{*}(S)$. Radó [5] has shown in this general case that $A^{*}(S) \leqq 2^{1 / 2} A(S)$. It has been shown (Huskey [3]) that if $f(x, y)$ is ACY on $Q_{0}$ then $A(S)=A^{*}(S)$. Theorems II and III show that somewhat more than this is true.

By the inequality

$$
A(S) \leqq \lim _{n} \inf A\left[P_{0}^{n}(f ; u, v)\right] \leqq \lim _{\alpha} \inf A\left[P_{0}^{n \alpha}(f ; u, v)\right]=A(S)
$$

we see that Theorem III implies Theorem II. However, independent proofs are given here. It is hoped that this presentation will encourage some reader to attack successfully the general case described above.

2. Preliminary. Let $Q(u, v)$ denote the square $u \leqq x \leqq u+1$, $v \leqq y \leqq v+1$. Let $D_{n}(u, v)$ denote a subdivision of $Q(u, v)$ into $n^{2}$ congruent squares. Let $S(u, v)$ denote the surface defined by $z=f(x, y)$, 
$(x, y) \in Q(u, v)$. Let $P_{n}(f ; u, v)$ denote the polyhedron inscribed in $S(u, v)$ and which corresponds to the subdivision $D_{n}(u, v)$ (see $\$ 1$ ). By boundary triangles of $P_{n}(f ; u, v)$ we mean triangles which have at least one vertex on the edge of $S(u, v)$.

LEMMA 1. The sum of the areas of the boundary triangles of $P_{n}(f ; u, v)$ or of $P_{0}{ }^{n}(f ; u, v)$ converge to zero as $n \rightarrow+\infty$. (See Huskey [3, p. 338].)

Due to periodicity $P_{n}(f ; u, v)$ can be replaced by a trianglewise identical polyhedron $P_{n}\left(f ; u^{\prime}, v^{\prime}\right)$ having only boundary triangles overlapping the edges of $S$ and whose other triangles are identical with those of $P_{0} n(f ; u, v)$. It is easy to show that $A[S(u, v)]=A(S)$, see Saks $[8$, p. 179]. Thus, in view of the above lemma, it is clear that Theorems I, II, and III will be established if we prove that, under the assumptions stated therein,

$\lim _{i} A\left[P_{n_{i}}(f ; u, v)\right]=A(S)$ for almost every $(u, v) \in Q_{0}$;

$\lim \inf _{n} A\left[P_{n}(f ; u, v)\right]=A(S)$ for almost every $(u, v) \in Q_{0}$; and

$\lim _{\alpha} A\left[P_{n_{\alpha}}(f ; u, v)\right]=A(S)$ for almost every $(u, v) \in Q_{0}$.

LEMma 2. If the measurable function $g_{n}(x, y)$ is periodic in $x$ of period $1 / n$ and, likewise, periodic in $y$ of period $1 / n$, then for any increasing sequence $\left\{n_{i}\right\}_{i=1}^{\infty}$ such that $n_{i}$ divides $n_{i+1}$ for all integers $i$, we have lim inf $g_{n_{i}}(x, y)=a$ constant for almost every $(x, y)$.

Proof. (This lemma seems to be well known but knowing of no convenient reference we give the following proof.) Let $\left\{n_{i}\right\}$ be given as described and let $\phi(x, y)=\lim _{\inf _{i}} g_{n_{i}}(x, y) . \phi(x, y)$ is periodic in $x$ and in $y$ of period $1 / n_{i}$ for every $i$. (1) We first assume $\phi(x, y)$ is continuous and it is easy to show that $\phi(x, y)$ is constant for all points $(x, y)$. (2) We now assume that $\phi(x, y)$ is summable on $Q_{0}$ and let $\phi_{h}(x, y)=h^{-2} \int_{0}^{h} \int_{0}^{h} \phi(x+u, y+v) d u d v . \phi_{h}(x, y)$ is continuous, periodic of period $1 / n_{i}$ in $x$ and in $y$ for all $i$, and is therefore a constant. $\phi_{h}(x, y) \rightarrow \phi(x, y)$ as $h \rightarrow 0$ for almost every $(x, y)$. Therefore, $\phi(x, y)$ is a constant almost everywhere. (3) To prove the theorem we note that $\phi(x, y)$ is measurable and let

$$
\phi_{N}(x, y)=\min [\max [\phi(x, y),-N], N] \quad \text { where } N \geqq 0 .
$$

Now $\left|\phi_{N}(x, y)\right| \leqq N$ is summable and periodic as above so $\phi_{N}(x, y)$ is a constant almost everywhere. $\phi_{N}(x, y) \rightarrow \phi(x, y)$ as $N \rightarrow+\infty$, so $\phi(x, y)$ is a constant almost everywhere. QED.

3. Proof of Theorem I. Let $h$ be a positive number and set

$$
f^{h}(x, y)=\frac{1}{h^{2}} \int_{0}^{h} \int_{0}^{h} f(x+\alpha, y+\beta) d \alpha d \beta
$$


which is an integral mean of $f(x, y)$. It is well known that $A\left(S^{h}\right) \rightarrow A(S)$ as $h \rightarrow 0$ where $S^{h}: z=f^{h}(x, y),(x, y) \in Q_{0}$ (see Saks [8, p. 179]).

Let $V_{c}{ }^{d}(x, f)$ denote the total variation of $f(x, y)$ for $x=x$ and $c \leqq y \leqq d . V_{a}^{b}(y, f)$ has a similar definition. It is easy to show (see Huskey [2, p. 251]; or Radó and Reichelderfer [6, p. 535]) that

$$
\int_{a}^{b} V_{c}^{d}\left(x, f-f^{h}\right) d x+\int_{c}^{d} V_{a}^{b}\left(y, f-f^{h}\right) d y \rightarrow 0 \quad \text { as } h \rightarrow 0 .
$$

Jessen [4] has shown that if $f(x)$ is summable and of period one in $x$ then for any increasing sequence of positive integers $\left\{m_{i}\right\}_{i=1}^{\infty}$ such that $m_{i}$ divides $m_{i+1}$ for all $i$ it is true that

$$
\lim \sum_{k=0}^{m_{i}-1}\left(1 / m_{i}\right) f\left(x+k / m_{i}\right)=\int_{0}^{1} f(x) d x
$$

for almost every $x$.

LEMMa 3.

$$
\begin{aligned}
& \left|A\left[P_{n}(f ; \xi, \eta)\right]-A\left[P_{n}\left(f^{h} ; \xi, \eta\right)\right]\right| \\
& \leqq \sum_{i, j=0}^{n-1} \frac{1}{n} V_{\eta+j / n}^{n+(j+1) / n}\left(\xi+\frac{i}{n}, f-f^{h}\right)+V_{\xi+i / n}^{\xi+(i+1) / n}\left(\eta+\frac{i}{n}, f-f^{h}\right) .
\end{aligned}
$$

The proof for Theorem 2.7 in Huskey [2, p. 253] can be easily altered to prove this lemma.

Using this lemma with Jessen's theorem and the fact that $A(S)$ is finite, we have for all $(\xi, \eta)$ not in a set $e_{1}$ of measure zero

$$
\begin{aligned}
\lim \sum_{k=0}^{m_{i}-1}\left(\frac{1}{m_{i}}\right) & {\left[V_{\eta}^{\eta+1}\left(\xi+\frac{k}{m_{i}}, f-f^{h}\right)+V_{\xi}^{\xi+1}\left(\eta+\frac{k}{m_{i}}, f-f^{h}\right)\right] } \\
& =\int_{\xi}^{\xi+1} V_{\eta}^{\eta+1}\left(x, f-f^{h}\right) d x+\int_{\eta}^{\eta+1} V_{\xi}^{\xi+1}\left(y, f-f^{h}\right) d y .
\end{aligned}
$$

Now, to prove the theorem, consider

$$
\begin{aligned}
\mid A(S)-A & {\left[P_{m_{i}}(f ; \xi, \eta)\right] \mid } \\
& \leqq\left|A(S)-A\left(S^{h}\right)\right|+\left|A\left(S^{h}\right)-A\left[P_{m_{i}}\left(f^{h} ; \xi, \eta\right)\right]\right| \\
& \quad+\left|A\left[P_{m_{i}}\left(f^{h} ; \xi, \eta\right)\right]-A\left[P_{m_{i}}(f ; \xi, \eta)\right]\right| .
\end{aligned}
$$

For $\epsilon>0$ given, choose $h$ so that the first term on the right of (7) is less than $\epsilon / 2$, and so that the right-hand side of (6) is less than $\epsilon / 4$. Next, choose $(\xi, \eta)$ in $Q_{0}-e_{1}$ and then choose $i$ so that the remaining two terms of (7) are less than $\epsilon / 2$. Note that 
$\lim A\left[P_{m_{i}}\left(f^{h} ; \xi, \eta\right)\right]=A\left(S^{h}\right)$ for every $(\xi, \eta)$ (see Saks [8, p. 177]). This completes the proof of Theorem $I$.

4. Proof of Theorem II. $A\left[P_{n}(f ; \xi, \eta)\right]$ is periodic in $\xi$ of period $1 / n$ and in $\eta$ of period $1 / n$. Therefore, except for $(\xi, \eta)$ in a set $e_{2}$ of measure zero, we have

$$
\lim _{i} \inf A\left[P_{m_{i}}(f ; \xi, \eta)\right]=A \text { (a constant) }
$$

where $m_{i}$ divides $m_{i+1}$ for all $i$.

By definition and periodicity of $A\left[P_{n}(f ; \xi, \eta)\right]$,

$$
\begin{aligned}
& \iint_{Q(\xi, \eta)}\left[T_{1}(f ; \alpha, \beta)+T_{2}(f ; \alpha, \beta)\right] d \alpha d \beta \\
& \quad=\sum_{D_{n}(\xi, \eta)} n^{2} \int_{0}^{1 / n} \int_{0}^{1 / n}\left(1 / n^{2}\right)\left[T_{1}\left(f ; x^{\prime}+\alpha, y^{\prime}+\beta\right)\right. \\
& \left.\quad+T_{2}\left(f ; x^{\prime}+\alpha, y^{\prime}+\beta\right)\right] d \alpha d \beta \\
& \quad=n^{2} \int_{0}^{1 / n} \int_{0}^{1 / n} A\left[P_{n}(f ; \xi+\alpha, \eta+\beta)\right] d \alpha d \beta \\
& \quad=\iint_{Q(\xi, \eta)} A\left[P_{n}(f ; \alpha, \beta) d \alpha d \beta .\right.
\end{aligned}
$$

This formula is essentially a result of Radó (see [5, p. 500]).

L. C. Young [9] has shown that if $f(x, y)$ is ACY on $Q_{0}$ then

$$
\iint_{Q_{0}} 2 T_{i}(f ; \alpha, \beta) d \alpha d \beta \rightarrow A(S), \quad i=1,2,
$$

as $n \rightarrow \infty$ in any manner (cf. $\$ 1$ ).

Now, almost everywhere in $Q_{0}$,

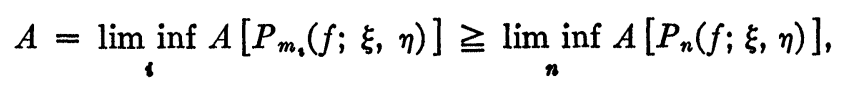

$n=1,2,3, \cdots$. So, using formulas (8), (9), and (10), the lemma of Fatou, and the property ACY of $f(x, y)$, we have for almost every $(\xi, \eta)$

$$
\begin{aligned}
A(S) & =\lim _{v} \iint_{Q_{0}} A\left[P_{m_{i}}(f ; \alpha, \beta)\right] d \alpha d \beta \geqq \int_{0}^{1} \int_{0}^{1} A d x d y \\
& \geqq \lim \inf A\left[P_{n}(f ; \xi, \eta)\right] \geqq A(S),
\end{aligned}
$$

which proves the theorem. 


\section{Proof of Theorem III.}

LeMma 4. If (a) E is a measurable set in the plane and of finite measure, (b) $\left\{g_{n}(x, y)\right\}_{n=0}^{\infty}$ is a sequence of non-negative real-valued functions measurable and summable on $E$, (c) lim $\inf _{n} g_{n}(x, y) \geqq g_{0}(x, y)$ almost everywhere on $E$, (d) lim $\sup _{n} \iint_{E} g_{n}(x, y) d x d y \leqq \iint_{E} g_{0}(x, y) d x d y$, then there exists a subsequence $\left\{g_{n_{i}}(x, y)\right\}_{i=1}^{\infty}$ such that $g_{n_{i}}(x, y) \rightarrow_{i} g_{0}(x, y)$ almost everywhere on $E$.

For a proof of this lemma see Radó and Reichelderfer [6, p. 551]. Using equations (4), (9), and (10) we readily see that $A\left[P_{n_{i}}(f ; \xi, \eta)\right]$ satisfies all the above conditions on $Q$ where $\left\{n_{i}\right\}$ is any sequence of integers such that $n_{i} \rightarrow \infty .\left(g_{\theta}(x, y)\right.$ corresponds to $A(S)$.) This completes the proof of Theorem III.

\section{REFERENCES}

1. A. S. Besicovitch, On the definition of the area of a surface by means of inscribed polyhedra, J. London Math. Soc. vol. 19 (1944) pp. 138-141.

2. H. D. Huskey, Contributions to the problem of Geöcze, Duke Math. J. vol. 10 (1943) pp. 249-257.

3. - Further contributions to the problem of Geöcze, Duke Math. J. vol. 11 (1944) pp. 333-339.

4. B. Jessen, On the approximation of Lebesgue integrals by Riemann sums, Ann. of Math. vol. 35 (1934) pp. 248-251.

5. T. Rad6, Some remarks on the problem of Geöcze, Duke Math. J. vol. 11 (1944) pp. 497-506.

6. T. Rad6 and P. Reichelderfer, Convergence in length and convergence in area, Duke Math. J. vol. 9 (1942) pp. 527-565.

7. P. Reichelderfer and L. Ringenberg, The extension of rectangle functions, Duke Math. J. vol. 8 (1941) pp. 231-242.

8. S. Saks, Theory of the integral, Warsaw, 1935.

9. L. C. Young, An expression connected with the area of a surface $z=F(x, y)$, Duke Math. J. vol. 11 (1944) pp. 43-57.

University of Pennsylyania 